\title{
Exposición fragmentada a la información periodística, polarización política y democracia representativa. El caso de los diputados del Parlamento catalán y su uso de los medios informativos
}

\author{
Iván LACASA MAS \\ Universitat Internacional de Catalunya \\ lacasa@uic.es \\ Olaf JANDURA \\ Heinrich-Heine-Universität Düsseldorf (Alemania) \\ jandura@uni-mainz.de \\ Francesc CANO CASTELLS \\ Universitat Internacional de Catalunya \\ fcano@uic.es
}

Recibido 12 de mayo de 2013

Aceptado: 10 de octubre de 2013

\begin{abstract}
Resumen
En no pocos países se viene discutiendo acerca del peligro democrático que supone una fragmentación del uso de los medios periodísticos. Al estudiar ese fenómeno, la academia ha centrado su atención en los ciudadanos, en detrimento de aquellos sujetos que, por pertenecer a la clase política, tienen una mayor capacidad de decisión e influencia. Gracias a una encuesta realizada a diputados del parlamento catalán hemos podido comprobar que entre ellos también se dan procesos de fragmentación informativa, debidos sobre todo a una evitación de información periodística y a una selección de medios guiada ideológicamente. Concluimos que, de manera análoga a como sucede con la población, la fragmentación del uso de los medios informativos puede llevar a una polarización de las élites políticas y, por tanto, a un deterioro del funcionamiento del sistema democrático representativo.

Palabras clave: democracia, polarización, fragmentación, medios de información, periodismo político, esfera pública
\end{abstract}

\section{Fragmentation, polarization and democracy.}

\section{The Catalan Parliament deputies and their news media use}

\begin{abstract}
In many countries scholars have discussed about the danger for the democracy posed by the fragmentation of the use of news media. In their study of this phenomenon, they have focused their attention on the citizens, to the detriment of the members of the political class. Thanks to a survey of members of the Catalan Parliament we have seen that politicians also have a tendency to fragmentation, mainly due to an avoidance of information and ideologically driven media selection. Our research concludes that, as in the case of the population, fragmentation of media use can lead to polarization of political elites and therefore to a deterioration of the representative democratic system.

Keywords: democracy, polarization, fragmentation, news media, political journalism, public sphere

\section{Referencia normalizada}

LACASA MAS, Iván; JANDURA, Olaf; y CANO CASTELLS, Francesc (2014): “Exposición fragmentada a la información periodística, polarización política y democracia representativa. El caso de los diputados del Parlamento catalán y su uso de los medios informativos". Estudios sobre el Mensaje Periodístico. Vol. 20, Núm. 1 (enero-junio), págs.: 431-450. Madrid, Servicio de Publicaciones de la Universidad Complutense.
\end{abstract}


Sumario: 1. Introducción. 2. Tipo ideal y tipo real de la exposición a contenidos periodísticos sobre política. 3. Método y operacionalización. 4. Resultados: uso de la oferta periodística por parte de los parlamentarios catalanes. 5. Conclusiones. 6. Referencias bibliográficas.

\section{Introducción}

Con los procesos de modernización social y de individualización de los modos de vida como trasfondo, el sistema político tiene frente a sí la difícil tarea de compatibilizar dos tareas básicas. Por un lado, fomentar al máximo una fuerte cohesión social, por el otro, garantizar que cada persona pueda recorrer con la máxima independencia un proyecto de vida propio (Beck y Sopp, 1997). En el sector de los medios de comunicación la búsqueda de ese equilibrio complejo fue encontrando su reflejo, a partir del final de la Segunda Guerra Mundial, en la aprobación y regulación progresivas de la emisiones privadas de radio y televisión en países que hasta entonces solo habían contado con radiodifusión pública (Trappel et al., 2011). En España, por ejemplo, este proceso recibió un impulso decisivo con la transición política a la democracia y se consagró cuando, a partir de 1989, se fundaron las primeras cadenas privadas de televisión (Barrera, 2004: 285-318; Palacio, 2001: 162-171). Las nuevas libertades de expresión y de información significaron un mayor pluralismo y una gran contribución a la sociedad y al sistema democrático (Prior, 2007), pero conllevaron también una exposición mucho más fragmentada de los ciudadanos a la información periodística, algo que colisiona con el tipo ideal de esfera pública que tiene el modelo de democracia liberal representativa (Ferree et al., 2002). Según ese modelo, la función que deben cumplir el periodismo es ejercer de foro: gracias a él los ciudadanos pueden disponer de todos los argumentos relevantes acerca de las diferentes cuestiones sociales y son capaces de elaborar un discurso común a partir del cual pueden llegar a formar posiciones mayoritarias (Ferree et al., 2002). En los últimos años, numerosos estudios han demostrado que una exposición fragmentada a la información conlleva una creciente polarización de la sociedad. Siguiendo la tradición marcada por la teoría de la exposición selectiva a la información periodística sobre política (Donsbach, 1991a, Donsbach, 1991b), diversas investigaciones han concluido que los ciudadanos son bien conscientes del partidismo de los medios y que se exponen preferentemente a las informaciones de aquellos a los que consideran más próximos a su propia posición política (Hollander, 2008). Debido a esa instrucción política unilateral, cada posición política ve cómo sus propios argumentos son los únicos que quedan confirmados diariamente, cosa que le lleva a reafirmarse aún más en ellos (Baum y Groeling, 2008: 360).

Teniendo todo ello en cuenta, y por razones de factibilidad del abordaje empírico del objeto de estudio, en el presente trabajo queremos centrar nuestra atención en un grupo social particular: las élites políticas, más concretamente, los miembros del Parlamento de Cataluña. Nos interesa saber si la teoría de la exposición selectiva es también válida para ellos, es decir, si el uso que hacen de los medios periodísticos es análogo al del ciudadano medio -en este caso de Cataluña- o si, por el contrario, la manera que esas élites políticas tienen de informarse les lleva a poder construir un mapa exhaustivo de los argumentos sobre los principales temas de discusión relevantes para la marcha del país. En la primera parte del artículo, con el modelo liberal-representa- 
tivo de esfera pública como telón de fondo, describiremos, por una parte, el tipo ideal $\mathrm{y}$, por otra, construiremos una imagen de la realidad de la exposición de los parlamentarios a la cobertura noticiosa política. Para dibujar esta imagen real haremos referencia a algunos estudios actuales, realizados en Estados Unidos, Europa y España. Concluiremos esa exposición formulando las tres preguntas de investigación que nos ayudarán a evidenciar el tipo de uso que los diputados del Parlamento catalán hacen de los medios de información. En la segunda parte del artículo expondremos la metodología aplicada en nuestro estudio y los resultados obtenidos. En la conclusión ligaremos las reflexiones teóricas sobre la democracia expuestas al inicio del artículo con los resultados de la encuesta hecha a los parlamentarios.

\section{Tipo ideal y tipo real de la exposición a contenidos periodísticos sobre política}

La política de medios que los sistemas democráticos llevan a cabo va dirigida a facilitar que el ciudadano pueda acceder, libre e independientemente, a información de calidad, de forma que quede establecido el fundamento de una esfera pública política que abarque a toda la sociedad (Gerhards y Neidhardt, 1991; Conde, 2002: 99-100). Dicha esfera pública es una categoría central en la imagen que de sí mismas tienen las democracias occidentales modernas, las cuales otorgan un valor eminente a la participación ciudadana en las decisiones políticas (Wendelin, 2011: 14). En los últimos años la esfera pública mediática ha alcanzado una consideración mayor que aquella esfera pública que nacía en los foros y las asambleas formalmente constituidas o que aquella que afloraba en los espacios donde las personas coincidían físicamente (Jarren y Donges, 2006: 121; Muñoz-Alonso y Rospir, 1999). Es este un hecho que puede explicarse, por una parte, atendiendo a la importancia fundamental que ha cobrado el periodismo en tanto que principal fuente de información sobre la actualidad y, por otra parte, atendiendo a la progresiva pérdida de relevancia de la esfera pública ligada a los foros y asambleas formales, algo que queda patente en la disminución del número de miembros de los partidos y sindicatos, así como en la creciente desafección política (Patterson, 1994; Rodríguez-Virgili et al., 2011; Liñeira y Pardos-Prado, 2009; Jordana, 1999). Cuanto más aumenta el diferencial de relevancia entre la esfera pública mediática y la esfera pública institucionalizada de los foros y las asambleas, más pertinente se vuelve la afirmación de Hartmut Weßler: si bien lo público no se agota en la esfera pública de los medios, hoy ya no se puede concebir una esfera pública completa sin estos últimos (Weßler, 1999: 23; Berrio, 2000: 162-167). Son muy elevadas, por tanto, las expectativas depositadas hoy en torno a la función social de los medios (Niedermayer, 2005). En tanto que equivalente funcional del asociacionismo y de los grupos de interés, se espera que sean instituciones que pongan a disposición un depósito común de temas y conocimientos que permita a los miembros de la sociedad intercambiar ideas y experiencias, sin importar demasiado que su origen sea de tipo mediado (Niedermayer, 2005).

Sin embargo, frente a esos objetivos se alza la realidad del uso que el público hace de la información. La creciente diversidad de la oferta ha traído consigo nuevas opciones y variantes de uso. Es en el mercado televisivo donde resulta más evidente que la proliferación de canales ha venido acompañada de un gran aumento del número de 
programas de entretenimiento, a los que el público ha otorgado su favor (Prior, 2007). Cuanto mayor es la oferta orientada al ocio, mayor es la probabilidad de que el público renuncie a consumir noticias y programas informativos (Ksiazek et al., 2010). En Estados Unidos, por ejemplo, el nuevo escenario se ha traducido en una pérdida masiva de audiencia por parte de las grandes cadenas de televisión establecidas.

Para responder a la pregunta de quiénes son los receptores que evitan exponerse a contenidos informativos es necesario profundizar en las características de la audiencia. Un bajo nivel de educación formal, bajos ingresos económicos o poco interés político han dado prueba de ser buenos predictores de una limitada exposición a los medios de información (Jandura y Meyen, 2010). Las personas que no tienen interés por la política tampoco aprovechan las múltiples oportunidades que se les ofrecen para obtener información acerca de ella (Bennett y Iyengar, 2008; Prior, 2007; Stroud, 2010).

A pesar de ser fruto de acercamientos teóricos muy variados, la conclusión que emerge de la mayoría de estudios que se centran en las características del receptor es que la creciente disparidad entre las capas sociales es un desafío para la comunicación en las sociedades democráticas. Las diferencias en los niveles de exposición a contenidos periodísticos sigue la tendencia general de una sociedad en la que las desigualdades verticales continúan ganando protagonismo frente a las horizontales.

Desde el punto de vista de la oferta mediática periodística es posible frenar una fragmentación de la esfera pública política mediante el fomento de una pluralidad real de los contenidos que los medios informativos ponen a disposición de los públicos. Cuando son varios los medios que, de manera auténticamente plural y completa, es decir, sin dejar de lado a ninguna de las principales opciones políticas, informan a los ciudadanos acerca de un mismo horizonte de hechos políticos, la base para un discurso que incluya a toda la sociedad y que genera un depósito común de temas y opiniones vuelve a quedar establecida (Dader, 2012). Para la construcción de una esfera pública democrática es indiferente la circunstancia concreta de cuáles son los medios periodísticos que consume la ciudadanía, siempre que esa ciudadanía que consume información sea mayoritaria y que esos medios informen de manera plural sobre los asuntos políticos. Diversos resultados procedentes de los estudios centrados en el comunicador llevan, sin embargo, a pensar que en España, igual que en Estados Unidos y en otros países, estamos frente a una polarización de la oferta informativa política (Rodríguez Díaz et al., 2012; De Miguel y Pozas, 2009; Berganza Conde et al., 2011; Farias Battle, 2011: 15-25, 104-105).

Otras investigaciones realizadas en Estados Unidos muestran que -muy especialmente en Internet, pero también en televisión- la cobertura informativa política animada por intereses partidistas ha aumentado considerablemente en los últimos años. La consecuencia es que se extiende un sesgo partidista que proviene de omitir la descripción de las posiciones y argumentos de los demás bandos políticos (Baum y Groeling, 2008), lo cual lleva a la instrucción unilateral del público. A la hora de analizar los estudios que parten de la realidad de una oferta fragmentada, no se puede pasar por alto el trabajo de Seymore-Ure, quien, ya en 1974, habló del efecto Press-Party-Paralellism (Seymour-Ure, 1974). Según ese autor, la línea editorial de un periódico -medida según el tenor de la valoración que hace de los políticos y partidos en sus comentarios 
o en sus recomendaciones de voto- se suele orientar hacia alguna de las posiciones de las fracciones prominentes, de forma que, en los países con sistemas multipartidistas, la cobertura mediática total tiende por fuerza a generar contenidos heterogéneos. Van Kempen recogió esta idea y, en 2007, publicó una aportación sobre el efecto MediaParty-Paralellism en la que comparó las tendencias políticas de la información televisiva y de prensa con la postura de los partidos relevantes en 15 países europeos (Van Kempen, 2007). Llegó a la conclusión de que en la mayoría de esos países se daba el efecto de Media-Party-Paralellism, el cual era más apreciable en la prensa diaria que en las noticias de televisión (Van Kempen, 2007: 314). Solo en los casos de Alemania, Finlandia e Irlanda fue imposible determinar un paralelismo entre las líneas editoriales y las posiciones de los principales partidos (Van Kempen, 2007: 309). En España, dicha relación está visiblemente consolidada (Hallin y Mancini, 2004).

A la vista de que los medios no logran generar un intercambio entre los diferentes bandos políticos, con frecuencia se ha expresado el pensamiento de que, mediante el discurso y la réplica, en la comunicación interpersonal sí se intercambian argumentos y, por tanto, sí se logra avanzar en la integración social. Sin embargo, esa afirmación está en contradicción con los hallazgos realizados en Estados Unidos. Mutz ha evidenciado que existe una tendencia a la "balkanization of interpersonal communication networks" (Mutz, 2006). Según él, las personas hablan de política con aquellos que defienden opiniones coincidentes. Ello hace que la probabilidad de incorporar nuevos matices a la visión que de un tema determinado se tiene quede muy reducida (Mutz, 2006).

Puede afirmarse, en resumen, que, igual que en Estados Unidos, también en España se está produciendo una polarización de la comunicación política. Es un fenómeno que se manifiesta en forma de:

1. Un sesgo -una distorsión partidista- en la cobertura informativa del campo político

2. Una exposición selectiva a contenidos periodísticos determinada por las convicciones políticas del público

3. Una comunicación interpersonal unilateral sobre temas políticos. Las consecuencias son que ha disminuido el entendimiento entre las diferentes posiciones políticas y que se ha tornado más difícil llegar a un consenso que permita abordar la resolución de los problemas sociales (Lee, 2007).

Cabe ahora preguntarse si la evolución que acabamos de describir es propia únicamente del común de la ciudadanía o si, por el contrario, también lo es de las élites políticas activas. La pregunta reza, entonces, si en los parlamentos, a pesar de ser instituciones llamadas a representar a una parte de la población lo más amplia posible, también es apreciable una polarización en el uso de los medios informativos (Pitkin, 1967). Varias encuestas realizadas a élites sociales han revelado que los políticos activos acuden a un repertorio de medios periodísticos mucho más amplio que el del ciudadano medio (Kepplinger y Marx, 2008; Slembeck, 1988). La cobertura mediática no tiene para ellos solo un sentido informativo general, también les ayuda a dar respuesta a los deseos y necesidades de su electorado. Es a través de los numerosos encuentros que tienen con los ciudadanos, pero también a través de los medios de comunicación, 
cómo los políticos conocen la realidad vital de aquellos a los que representan. Esto es especialmente válido para la exposición a los medios de comunicación regionales. Gracias al consumo de periódicos regionales, así como de emisoras de radio y canales de televisión regionales, los políticos permanecen atentos a los acontecimientos que suceden en su circunscripción electoral y se informan sobre sus problemas más actuales. Las diferentes encuestas realizadas a miembros de parlamentos alemanes y suizos dieron como resultado que el tiempo que estos dedicaban a la lectura de periódicos era entre dos y tres veces más largo que el del ciudadano medio y que, además de leer diferentes periódicos suprarregionales, concebían a la lectura de periódicos regionales como una de sus "obligaciones" diarias (Kepplinger y Marx, 2008; Slembeck, 1988; Puhe y Würzberg, 1989; Peter, 1998). Así era como se aseguraban de, por un lado, estar al día de los aconteceres en sus circunscripciones electorales y de, por el otro, acumular una diversidad de perspectivas que les permitiera afrontar con garantías de éxito los retos políticos que fueran apareciendo en el horizonte.

En el ya mencionado estudio de Van Kempen (2007) sobre el Media-Party-Paralellism quedó determinado que el paisaje mediático español poseía una fuerte tendencia ideológica. De los hallazgos de diferentes estudios se desprende que los cuatro grandes periódicos españoles -El País, El Mundo, ABC y La Razón- cubren uniformemente, de izquierda a derecha, todas las posiciones políticas relevantes (Berganza Conde et al., 2011; De Miguel y Pozas, 2009; López García, 2009; Cortiñas Rovira y Pont Sorribes, 2009). Los lectores de El País, por ejemplo, esperan recibir y reciben sus noticias y argumentos políticos desde la perspectiva de una izquierda liberal, mientras que los lectores de $A B C$ cultivan una posición política conservadora. En Alemania, donde también cuatro periódicos cubren el espectro de posiciones y argumentos políticos relevantes, los análisis de contenido y las encuestas a expertos (políticos, altos funcionarios o líderes empresariales) han conducido a conclusiones análogas acerca de las líneas editoriales de los periódicos (Kepplinger, 1998). La experiencia alemana indica, pues, que las encuestas a políticos pueden ser oportunas para situar a las diferentes ofertas mediáticas en un espectro político.

Sobre la base de las reflexiones que hemos expuesto en este apartado, deseamos responder a las siguientes preguntas de investigación:

1. ¿Qué amplitud tiene la exposición informativa de los diputados del Parlamento de Cataluña?

2. ¿Cómo evalúan los diputados la posición política de los diferentes medios periodísticos presentes en Cataluña?

3. ¿Consumen los diputados medios que confirman su propia posición política o, por el contrario, medios que cubren todo el espectro político del país?

\section{Método y operacionalización}

Para responder a estas preguntas de investigación se realizó, de mayo a septiembre de 2011, una encuesta cuantitativa entre los diputados del Parlamento catalán de la IX Legislatura. Las preguntas se realizaron en catalán. El cuestionario constó de cinco bloques temáticos diferentes. 
En el primer bloque, centrado en el uso de los medios periodísticos, se les pidió a los diputados que dijesen si consumían -y con qué frecuencia- un total de 43 ofertas de información diferentes, entre ellas 6 periódicos suprarregionales, 5 periódicos regionales y 9 locales, 9 cadenas de televisión suprarregionales y 2 regionales, 4 emisoras de radio suprarregionales y 5 regionales, además de 5 cadenas informativas de radio o televisión. A fin de evitar la fatiga durante la cumplimentación, las preguntas sobre cada uno de los tipos de medio se repartieron en diversos bloques a lo largo del cuestionario. La frecuencia de uso se fijó mediante las categorías diariamente, tres $o$ cuatro veces por semana, una o dos veces por semana y raras veces. ${ }^{1}$

Para las ofertas informativas más de referencia o con mayor audiencia, en un segundo paso se pidió a los diputados que juzgasen la orientación de cada una de ellas y que la ubicaran en un espectro político-mediático que iba desde la extrema izquierda (valor de escala 1) a la extrema derecha (valor de escala 10). Cuanto menor fue el valor que los diputados otorgaron a un medio determinado, más a la izquierda del espectro quedó clasificado este; cuanto mayor fue ese valor, más conservadora se juzgó que era la línea editorial del medio periodístico correspondiente.

En el tercer bloque relevante para el presente estudio los diputados indicaron datos sociodemográficos sobre su propia persona (edad, género, formación) y sobre su carrera política (adscripción, posicionamiento propio en el espectro político). También se les preguntó por su postura respecto a una posible independencia de Cataluña y por su valoración de la cobertura informativa que los medios más relevantes habían hecho de ese tema político. Asimismo, se pronunciaron sobre el consumo de medios online que hacían y sobre su uso de nuevas posibilidades de comunicación, tales como Twitter y Facebook. En la evaluación de datos que haremos en este artículo no podremos tener en cuenta todas estas cuestiones.

De los 135 miembros del Parlamento de Cataluña, 99 rellenaron el cuestionario, lo que significa un índice de respuesta muy elevado: $73 \%$. Otros estudios comparables -realizados, por ejemplo, en Alemania- alcanzaron solo una cuota de entre el $7 \%$ y el $39 \%$, según la fracción política de que se tratara (Kepplinger y Marx, 2008). En nuestro caso la alta tasa se alcanzó, en gran parte, gracias a una intensa comunicación in situ con los diputados. Así, por ejemplo, se contactó directamente con aquellos que todavía no habían rellenado el cuestionario y se les volvió a pedir que participaran. A pesar de este acercamiento personalizado, en todo momento se garantizó el anonimato de los encuestados. Si se observa el índice de respuesta de los diferentes parti-

${ }_{1}^{1}$ Los medios por los que se les preguntó fueron los siguientes: ABC, Ara, El Mundo, El País, El Periódico de Cataluña, El Punt-Avui, La Gaceta, La Razón, La Vanguardia, Público, Bon dia Lleida, Diari de Girona, Diari De Sabadell, Diari de Tarragona, Diari de Terrassa, El Punt (edición Girona), La Mañana, Regió 7, Segre, Cadena Ser, Catalunya Ràdio, COM Ràdio, COPE, ONA FM, Onda Cero, Punto Radio, RAC1, Radio Nacional, Ràdio 4, Catalunya Informació, Radio Intereconomía, Radio 5 TN, Antena 3, Cuatro, Intereconomía, La Sexta, La 1, La 2, Telecinco, TV3, 8TV, Canal 3/24, Canal 24 Horas. Tanto en prensa, como en televisión y radio se incluyeron campos de respuesta libre para que los parlamentarios pudieran incluir medios que no estuvieran en el listado (locales o internacionales, por ejemplo). 
dos políticos, se advierte que solo el índice de respuesta del Partido Popular -todavía con un considerable 39\%- se aparta de la alta tasa de respuesta obtenida. Esta menor disposición a participar puede ser atribuida a la carga de trabajo que recaía sobre los diputados de ese partido, debido a su condición de socio minoritario de un acuerdo de estabilidad parlamentaria con el partido de gobierno (Tabla 1).

Tabla 1: Índice de respuesta a la encuesta. Pregunta: A quin grup parlamentari pertany? (¿A qué grupo parlamentario pertenece?). Fuente: elaboración propia.

\begin{tabular}{|c|c|c|c|}
\hline $\begin{array}{l}\text { Grupo } \\
\text { parlamentario }\end{array}$ & $\begin{array}{c}\mathrm{N}^{\mathrm{o}} \\
\text { (diputados) }\end{array}$ & $\begin{array}{c}\mathrm{N}^{\mathrm{o}} \\
\text { (encuestados) }\end{array}$ & $\begin{array}{c}\text { Índice de } \\
\text { respuesta }(\%)\end{array}$ \\
\hline$\overline{\mathrm{CiU}}$ & 62 & 48 & 77 \\
\hline PSC & 28 & 22 & 79 \\
\hline PP & 18 & 7 & 39 \\
\hline ICV-EUiA & 10 & 10 & 100 \\
\hline ERC & 10 & 8 & 80 \\
\hline SI & 3 & 2 & 67 \\
\hline C's & 3 & 2 & 67 \\
\hline TOTAL & 135 & 99 & 73 \\
\hline
\end{tabular}

La edad media de los diputados encuestados fue de 47 años. El encuestado más joven tenía 23 años, el más mayor, 69. El 54\% fueron hombres y el $46 \%$ mujeres. Casi todos los diputados (86\%) poseían una educación formal muy alta, es decir, formaban parte de la élite educativa del país. El 11\% tenía una educación formal media y solo el 3\% una educación formal baja. En lo que a la propia valoración política se refiere, la distribución quedó ligeramente desplazada hacia la izquierda. En una escala de 10 puntos, en la que el valor 1 era la posición más a la izquierda y el valor 10 la posición más a la derecha, el valor medio fue de 4,2. Quienes se posicionaron más a la izquierda fueron los diputados de ICV-EUiA, con un valor medio de $2,7^{\mathrm{a}}$, seguidos por el Grupo Mixto SI (3,0 $\left.0^{\text {ab }}\right)$, ERC (3, $\left.5^{\text {ab }}\right)$, Grupo Mixto C's $\left(3,5^{\text {ab }}\right)$ y el PSC $\left(3,5^{\text {ac }}\right)$. Los diputados de CiU se ubicaron a sí mismos algo más a la derecha del valor medio $\left(4,8^{\mathrm{ab}}\right)$. Como era de esperar, los políticos del PP mostraron la valoración más conservadora, con un 6,0 ( ${ }^{\mathrm{bc}}$ ) (UNIANOVA $\mathrm{p}<0.001$ ).

Para examinar la calidad de nuestra encuesta, contrastamos sistemáticamente los perfiles personales de todos los parlamentarios, disponibles en la web oficial del Parlamento de Cataluña. La comparación evidencia que nuestra encuesta no contiene errores estructurales que afecten a alguno de los partidos o grupos políticos. Hemos trabajado, por tanto, con datos que reflejaban bien la realidad del Parlamento catalán de la IX Legislatura. Las distribuciones de las variables de edad, género y procedencia de nuestros encuestados se corresponden en lo esencial con las estadísticas del Parlamento (Tabla 2). Acerca del nivel de estudios formales de los diputados, no existe ninguna estadística parlamentaria oficial con la que pudiéramos comparar nuestros datos².

2 Tampoco constaba en los perfiles personales de los políticos disponibles en www.parlament.cat su lugar de nacimiento. Solo aparecía la circunscripción por la que se habían presentado como candidatos. Ese es el dato que hemos podido utilizado para contrastar nuestra encuesta. 
Puede comprobarse que, en lo que a las variables de edad y género se refiere, el Parlamento representa muy bien a la población catalana. Sin embargo, en lo que respecta a la educación formal, se constata que -como en muchos otros parlamentos europeoslos diputados provienen en su mayoría de la élite educativa de la sociedad. El hecho de que Barcelona esté infrarrepresentada en el Parlamento ha de atribuirse a la normativa interna de esa institución, la cual, a fin de promover un equilibrio territorial, establece un número fijo de diputados para cada circunscripción electoral: 85 para Barcelona, 17 para Girona, 15 para Lleida y 18 para Tarragona. En comparación con la distribución geográfica real de la población catalana, las tres últimas circunscripciones están sobrerrepresentadas.

Tabla 2: Datos sociodemográficos comparados. Fuente: elaboración propia

\begin{tabular}{|c|c|c|c|}
\hline Variables & $\begin{array}{c}\text { Estadística de los } \\
\text { encuestados \% }\end{array}$ & $\begin{array}{l}\text { Estadística del } \\
\text { Parlamento \% }\end{array}$ & $\begin{array}{c}\text { Población } \\
\text { catalana \% }\end{array}$ \\
\hline Sexo: Hombre & 54,0 & 59 & 49 \\
\hline Edad & 47,4 & 47 & 41 \\
\hline \multicolumn{4}{|l|}{ Educación $^{3}$} \\
\hline SIN ESTUDIOS & 0,0 & $\mathrm{x}$ & 11 \\
\hline ESTUDIOS PRIMARIOS & 3,0 & $\mathrm{x}$ & 37 \\
\hline $\begin{array}{l}\text { ESTUDIOS } \\
\text { SECUNDARIOS }\end{array}$ & 11,0 & $\mathrm{x}$ & 35 \\
\hline ESTUDIOS SUPERIORES & 86,0 & $\mathrm{x}$ & 18 \\
\hline \multicolumn{4}{|l|}{ Teritorio } \\
\hline Barcelona & 61,0 & 62,0 & 73,0 \\
\hline Tarragona ${ }^{4}$ & 15,0 & 13,0 & 11,0 \\
\hline Lleida $^{5}$ & 11,0 & 11,0 & 10,0 \\
\hline Girona $^{63}$ & 13,0 & 13,0 & 6,0 \\
\hline
\end{tabular}

\section{Resultados: uso de la oferta periodística por parte de los parlamentarios ca- talanes}

Vamos a realizar la evaluación de los resultados de la encuesta en tres pasos. Vienen dictados por las preguntas de investigación que hemos definido más arriba. En primer lugar estudiaremos el alcance del consumo de medios informativos que hacen los parlamentarios catalanes.

Los datos muestran que los diputados hacen un seguimiento intensivo de la oferta informativa mediática. La radio, con un valor del $78 \%$, y los periódicos regionales y suprarregionales, con un $76 \%$, tienen para ellos un valor mucho más alto que para el resto de la población catalana. Por el contrario, el consumo de televisión informativa

${ }^{3}$ No hay datos oficiales disponibles

${ }^{4}$ Comarcas que integran la provincia de Tarrgaona: Montsià, Baix Ebre, Terra Alta, Ribera d'Ebre, Priorat, Baix Camp, Alt Camp, Tarragonès, Conca de Barberà, Baix Penedès

5 Comarcas que integran la provincia de Lleida: Vall d'Aran, Alta Ribagorça, Pallars Sobirà, Pallars Jussà, Alt Urgell, Noguera, Solsonès, La Segarra, Urgell, Pla d'Urgell, Les Garrigues, Segrià

${ }^{6}$ Comarcas que integran la provincia de Girona: La Selva, Gironès, Baix Empordà, Alt Empordà, Pla de l'Estany, Garrotxa, Ripollès, Cerdanya 
es más alto entre la población que entre los diputados (91\% frente a $66 \%)$. En la dieta mediática diaria de los parlamentarios figura, además, la prensa local, que es leída por el $60 \%$ de ellos, y la prensa internacional (10\%). Su uso mediático queda completado por una fuerte preferencia hacia la oferta de información política televisiva y radiofónica, una oferta que tiene poca relevancia en el caso de la población catalana (Tabla 3).

Tabla 3: Consumo de medios de los diputados y de la población ${ }^{7}$.

Fuente: elaboración propia.

\begin{tabular}{|l|r|r|l|r|r|}
\hline \multicolumn{3}{|c|}{ Cada día (Sí/No) } & \multicolumn{3}{c|}{ Habitualmente (Sí/ No) } \\
\hline Medios & $\begin{array}{r}\text { Diputados } \\
\mathbf{\%}\end{array}$ & $\begin{array}{r}\text { Población } \\
\mathbf{\%}\end{array}$ & Medios & $\begin{array}{r}\text { Diputados } \\
\%\end{array}$ & $\begin{array}{r}\text { Población } \\
\%\end{array}$ \\
\hline Radio & 78 & 58,0 & $\begin{array}{l}\text { Televisión } \\
\text { información }\end{array}$ & 99 & 18,15 \\
\hline Periódicos & 76 & 35,7 & $\begin{array}{l}\text { Radio } \\
\text { información }\end{array}$ & 95 & 2,90 \\
\hline Televisión & 66 & 92,0 & $\begin{array}{l}\text { Televisión } \\
\text { local }\end{array}$ & 52 & $0,2^{*}$ \\
\hline Periódicos locales & 60 & 10,1 & Radio local & 37 & $0,3^{*}$ \\
\hline $\begin{array}{l}\text { Periódicos } \\
\text { internacionales }\end{array}$ & 10 & sin datos & $\begin{array}{l}\text { Televisión } \\
\text { internacional }\end{array}$ & 29 & sin datos \\
\hline
\end{tabular}

* No existen datos fiables, debido a la falta de medios económicos o técnicos para realizar las mediciones.

Estas cifras confirman los resultados de otras encuestas dirigidas a políticos, las cuales ya habían mostrado cómo, en las democracias parlamentarias representativas, estos hacen un uso de medios muy amplio, sobre todo comparado con el del resto de ciudadanos (Slembeck, 1988; Puhe y Würzberg, 1989; Peter, 1998). No cabe olvidar que para los representantes parlamentarios la cobertura informativa periodística es tanto una fuente de información sobre la circunscripción electoral a la que representan, como un punto de referencia para valorar su mismo trabajo, pues refleja su propia actividad política y de la de los partidos que con él compiten. Un uso amplio de diferentes tipos de medios -prensa, televisión, radio, etc.- ayuda a los políticos a orientarse.

El consumo de medios noticiosos que acabamos de exponer nos ha servido de base para elaborar una tipología de los diferentes perfiles de uso que predominan entre los diputados. La diferenciación en cuatro estilos diferentes de informarse se ha mostrado como mejor resultado estadístico de un análisis de clúster (kmeans, $\mathrm{F}=268,7, \mathrm{p}<$ $0.001)$ :

1. Politicos regionalistas y suprarregionalistas (24\%): quienes cultivan este estilo se exponen en la misma medida que el resto de políticos a la radio y la televisión suprarregionales, así como a los canales de noticias televisivos y radiofónicos. Sin embargo, evitan las ofertas de información local e internacional (diarios locales e internacionales o radio local).

7 Los datos de la población provienen del EGM de febrero de 2012 y del Baròmetre de la Comunicació i Cultura de marzo de 2012. 
2. Políticos de prensa (52\%): los diputados que pertenecen a este perfil de consumo se informan, más que la media, a través de toda la oferta informativa impresa. Recurren, en mucha mayor medida que el resto de perfiles, a la prensa local, regional y suprarregional. Por contra, para ellos la información televisiva juega un papel menor.

3. Políticos radiotelevisivos (14\%): los parlamentarios que pertenecen a esta categoría se caracterizan por considerar a la televisión -tanto a nivel local, como regional y suprarregional- la fuente de información más importante; a la radio, como la segunda más importante. En su dieta informativa, los diarios locales e internacionales no juegan ningún papel (o solo uno muy reducido).

4. Políticos superinformados (10\%): se distinguen por consumir de forma muy intensiva todo tipo de medios, tanto de ámbito local, como regional, nacional e internacional. Este perfil de acceso a la información se caracteriza, sobre todo, por consumir por encima de la media no solo radio y televisión local, sino también prensa y espacios informativos de cadenas internacionales de televisión.

Tabla 4: Perfil de consumo de medios de información de los políticos del Parlamento de Cataluña. Fuente: elaboración propia.

\begin{tabular}{|c|c|c|c|c|c|c|}
\hline Tasa de respuesta afirmativa ("sí") & 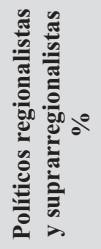 & 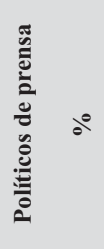 & ब゚ & 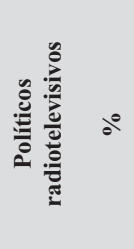 & है & $0^{\circ}$ \\
\hline $\begin{array}{l}\text { Uso habitual de canales informativos de } \\
\text { televisión (regional y suprarregional) }\end{array}$ & 100 & 100 & 90 & 100 & 99 & \\
\hline $\begin{array}{l}\text { Uso habitual de emisoras informativas de } \\
\text { radio }\end{array}$ & 96 & 96 & 80 & 100 & 95 & \\
\hline $\begin{array}{l}\text { Uso diario de información en emisoras de } \\
\text { radio (regionales y suprarregionales) }\end{array}$ & 75 & 79 & 80 & 79 & 78 & \\
\hline $\begin{array}{l}\text { Uso diario de prensa (regional y } \\
\text { suprarregional) }\end{array}$ & 58 & 89 & 60 & 71 & 76 & \\
\hline $\begin{array}{l}\text { Uso diario de información en cadenas de } \\
\text { televisión (regionales y suprarregionales) }\end{array}$ & 67 & 70 & 10 & 93 & 66 & \\
\hline Uso diario de prensa local & 4 & 100 & 60 & 0 & 59 & \\
\hline $\begin{array}{l}\text { Uso habitual de información en cadenas } \\
\text { de televisión }\end{array}$ & 50 & 40 & 90 & 71 & 51 & \\
\hline $\begin{array}{l}\text { Uso habitual de información en emisoras } \\
\text { de radio local }\end{array}$ & 0 & 26 & 90 & 100 & 37 & \\
\hline $\begin{array}{l}\text { Uso habitual de información en cadenas } \\
\text { internacionales de televisión }\end{array}$ & 38 & 15 & 90 & 21 & 29 & \\
\hline Uso diario de periódicos internacionales & 4 & 13 & 10 & 7 & 10 & \\
\hline
\end{tabular}

La pertenencia a uno de estos perfiles no depende de la adscripción política, de la edad o del sexo. Más bien muestra que, en lo tocante al tipo de medio (prensa, televisión, radio, etc.), dentro de las fracciones parlamentarias -o también de las franjas de edad de los diputados, por ejemplo- conviven varios estilos de información. La tipo- 
logía que acabamos de presentar deja claro que hay grandes diferencias en el consumo de ofertas de información regionales y suprarregionales. Vamos a examinarlas. Para ello hemos clasificado las más de 40 ofertas periodísticas estudiadas en dos categorías básicas: medios editados por empresas con sede en Madrid y medios editados por empresas con sede en alguna de las circunscripciones de Cataluña. Entre los primeros se cuentan La Gaceta, La Razón, ABC, El Mundo, El País, COPE, Onda Cero, Punto Radio, Radio Nacional, Cadena Ser, Intereconomía, Antena 3, La 1, La 2, Telecinco, Cuatro y La Sexta. Entre los segundos, El Periódico, La Vanguardia, Ara, El Punt-Avui (edición Girona), Público ${ }^{8}$, Ràdio 4, COM Ràdio, Catalunya Ràdio, Racl, 8TV y TV3.

Un primer recuento de los medios utilizados regularmente (mínimo 3 veces por semana) muestra que, en promedio, 7,1 de las 17 ofertas suprarregionales y 7,0 de las 13 regionales están presentes en el espectro mediático de los diputados. Las ofertas utilizadas más a menudo pertenecen al ámbito regional. TV3 (96\%) y Catalunya Informació (91\%), dos medios regionales, figuran entre las fuentes de información más importantes para los diputados. Les siguen Catalunya Ràdio (71\%), La Vanguardia (67\%) y $R A C 1$ (59\%). La oferta centrada en España es seguida por un tercio amplio de los diputados (El País, 36\%; La 1, 34\%; Cadena SER, 30\%).

Tal como hemos visto más arriba, uno de los rasgos que caracterizan al mercado de los medios en España es una polarización política de la oferta en un nivel regional y otro suprarregional (Van Kempen, 2007). Por ello, en la segunda fase de nuestro estudio vamos a comprobar la valoración que los parlamentarios hacen de la línea editorial de los medios editados por empresas con sede en Barcelona u otra circunscripción de Cataluña (regionales) y de los editados en Madrid (suprarregionales). Lo vamos a hacer centrándonos en el caso de la prensa9. A los diputados se les pidió que ubicaran a los periódicos que leían habitualmente en una escala de izquierda a derecha.

El siguiente gráfico muestra que los políticos son bien capaces de otorgar una línea editorial a los diarios relevantes. Al diferenciar los diarios suprarregionales de los regionales, se percibe que los periódicos suprarregionales El País, El Mundo, $A B C, L a$ Razón y La Gaceta amplían el espectro político-mediático catalán. A nivel regional, este queda acotado por Público, El Periódico, ARA, El Punt-Avui (edición Girona) y La Vanguardia. La comparación de ambos espectros revela que el posicionamiento percibido de los periódicos regionales se encuentra más a la izquierda que la de los periódicos suprarregionales.

8 Consideramos a Público un caso excepcional en el panorama de medios regionales, puesto que su línea editorial poseyó una clara vocación de ámbito suprarregional.

9 Para las emisoras de radio y los canales de televisión obtuvimos resultados muy semejantes. Por la limitación de espacio no vamos a poder exponerlos aquí. 


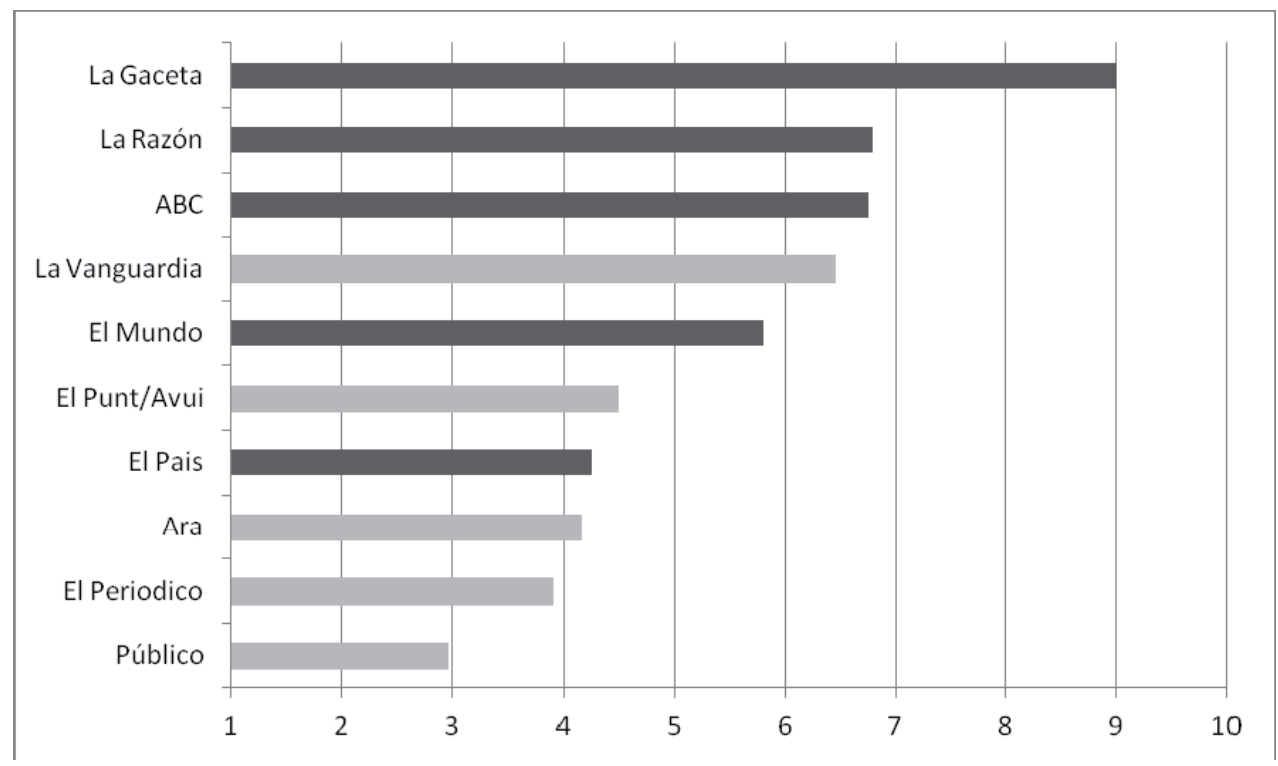

Gráfico 1: Listado de los medios más leídos por los parlamentarios catalanes, ubicados en una escala izquierda-derecha (1, extrema izquierda; 10, extrema derecha). Fuente: elaboración propia.

Número de casos: ABC, 4; Ara, 47; El Mundo, 10; El País, 36; El Periódico, 48; El Punt-Avui (edición Girona), 46; La Gaceta, 1; La Razón, 5; La Vanguardia, 72; Público, 21

Los resultados muestran con claridad que los diputados catalanes son capaces de ubicar el posicionamiento político de los medios periodísticos suprarregionales más importantes de forma clara y diferenciada en un espectro izquierda-derecha. Las respuestas permiten apreciar fácilmente que -sin darle ese nombre- los diputados son conscientes de la existencia del efecto Media-Party-Paralellism, descrito por van Kempen para el caso de España y de otros países (Van Kempen, 2007). Saben juzgar sin problemas qué oferta informativa regional y suprarregional queda más próxima a qué partido, es decir, qué medio da soporte político-informativo a qué partido. E1 92\% de los diputados está convencido de que La Vanguardia se corresponde más estrechamente con la posición política de CiU. Asimismo identifican al Ara con ERC (77\%), a El País (88\%) y El Periódico (94\%) con el PSC. Por último, la línea editorial de Público es juzgada por el $70 \%$ de los encuestados como la más cercana a ICV (Tabla 5).

Desde las afirmaciones de que (1) los diputados disponen de un repertorio de medios numéricamente amplio y (2) conocen muy bien el posicionamiento político de los medios, es fácil abordar nuestra tercera pregunta de investigación: ¿utilizan los diputados preferentemente la oferta periodística que apoya su propio posicionamiento político o, por el contrario, siguen a medios adscribibles a cualquier punto del espectro político? Para responder a este interrogante volveremos a diferenciar entre el caso de los medios regionales (editados por empresas con sede en Barcelona u otra de las cir- 
cunscripciones de Cataluña) y el de los suprarregionales (editados por empresas con sede en Madrid) ${ }^{10}$.

El consumo que los diputados hacen de las ofertas informativas mediáticas regionales muestra que hay medios regionales, como La Vanguardia o TV3, a los que todos ellos acuden, independientemente de su adscripción política. Esos medios periodísticos líderes disfrutan de la oportunidad de presentar los diferentes aspectos y opiniones sobre la actualidad política y social a un público no solo amplio, sino -véase el caso de los políticos- también influyente. Por ambos motivos, desde la teoría democrática, cabe otorgarles una función de integración especialmente importante. Ciertamente, la fragmentación de la exposición de los políticos a ofertas regionales de información se da solo en un segundo nivel de consumo, tampoco nada despreciable. Así, por ejemplo, la Cadena Ser es escuchada principalmente por los diputados del PSC (el 85\% de ellos lo hace), RACl por los diputados de CiU, Catalunya Ràdio sobre todo por los diputados de ICV y ERC ${ }^{11}$. Algo semejante sucede en el ámbito de la prensa. En él se da una fuerte correlación entre la clasificación política que hace de sí mismo el diputado y el posicionamiento de la línea editorial en el espectro político-mediático de los diarios que lee. El Ara, por ejemplo, es leído principalmente por los miembros de CiU, ICV y ERC, mientras que El Periódico encuentra su público entre los parlamentarios del PSC, ICV y ERC (Tabla 5)

Tabla 5: Media-Party-Parallelism ${ }^{12}$. Fuente: elaboración propia.

\begin{tabular}{|l|c|c|c|c|c|r|}
\hline \multicolumn{1}{|c|}{ Medios } & $\begin{array}{c}\text { Todos } \\
\mathbf{\%}\end{array}$ & $\begin{array}{c}\mathbf{C i U} \\
\mathbf{\%}\end{array}$ & $\begin{array}{c}\text { PSC } \\
\mathbf{\%}\end{array}$ & $\begin{array}{c}\text { PP } \\
\mathbf{\%}\end{array}$ & $\begin{array}{c}\text { ICV } \\
\mathbf{\%}\end{array}$ & $\begin{array}{c}\text { ERC } \\
\mathbf{\%}\end{array}$ \\
\hline TV3 (TV) & 97 & 94 & 91 & 85 & 100 & 100 \\
\hline Catalunya Ràdio (radio) & 71 & 67 & 68 & 29 & 90 & 100 \\
\hline La Vanguardia (prensa) & 67 & 92 & 59 & 71 & & \\
\hline RAC1 (radio) & 59 & $\mathbf{8 1}$ & 31 & 57 & 10 & 62 \\
\hline Ara (prensa) & 52 & $\mathbf{5 7}$ & & & $\mathbf{6 0}$ & $\mathbf{1 0 0}$ \\
\hline El Periódico (prensa) & 42 & & 77 & & 70 & 75 \\
\hline El País (prensa) & 38 & & $\mathbf{8 6}$ & & $\mathbf{7 0}$ & \\
\hline La 1 (TV) & 34 & 17 & $\mathbf{5 9}$ & 42 & 40 & 40 \\
\hline
\end{tabular}

${ }^{10}$ Como orientación, indicamos, a grandes rasgos, cuál era la composición de los diferentes grupos mediáticos en el momento de hacer la encuesta: RTVE (La 1, La 2, Canal 24 horas, RNE, Radio 4, Radio 5), CCMA (TV3, Canal 33, Canal 3/24, Catalunya Ràdio, Catalunya Informació), Grupo Godó (La Vanguardia, 8TV, RAC1), Grupo Zeta (El Periódico, ONA FM), Prisa (El País, Cuatro, Cadena SER), Planeta (La Razón, Antena 3, Onda Cero), Mediaset (Tele 5), Intereconomía (La Gaceta, Intereconomía TV, Radio Intereconomía), MediaPro (Público, La Sexta), Conferencia Episcopal Española y diócesis españolas (COPE), ABC ( $A B C, A B C$ Punto Radio).

${ }^{11}$ Puede ser oportuno recordar en este punto que, en el momento en que hicimos la encuesta, el Gobierno de la Corporació Catalana de Mitjans Audiovisuals (CCMA) estaba presidido, a propuesta de ERC, por el Dr. Enric Marín.

${ }^{12}$ La tabla contiene una selección de los casos que, por porcentaje, hemos considerado más relevantes. Dejamos la casilla en blanco en aquellos casos en que este era inexistente o muy bajo (inferior a un $10 \%$ ) 


\begin{tabular}{|l|r|r|r|r|r|r|}
\hline Cadena SER (radio) & 30 & & $\mathbf{8 1}$ & 29 & 30 & 13 \\
\hline COM Ràdio (radio) & 21 & 10 & 37 & 14 & 50 & 29 \\
\hline 8TV (TV) & 14 & 23 & & & & \\
\hline La Sexta (TV) & 13 & & 27 & & 40 & \\
\hline El Mundo (prensa) & 12 & & & $\mathbf{8 5}$ & & \\
\hline Antena 3 (TV) & 10 & & & $\mathbf{5 7}$ & & \\
\hline Cuatro (TV) & 8 & & 18 & & & \\
\hline La Razón (prensa) & 5 & & & $\mathbf{5 7}$ & & \\
\hline Intereconomía $(\mathrm{TV})$ & 2 & & & $\mathbf{2 9}$ & & \\
\hline
\end{tabular}

Entre los medios periodísticos suprarregionales no encontramos ninguno que, por grado de consumo entre los parlamentarios, pueda ser considerado tan central como podían serlo, a nivel regional, TV3 o La Vanguardia, por ejemplo. El fenómeno, aparentemente paradójico, de que casi cada político usa un medio suprarregional y de que, a la vez, entre ellos la exposición a ese tipo de medio es relativamente baja, puede encontrar una explicación plausible en el uso fragmentado de la oferta informativa suprarregional que quizá estén haciendo. Queda claro, antes de todo, que los políticos que pertenecen a partidos de matriz española consumen con mucha más frecuencia medios suprarregionales que los políticos adscritos a partidos de matriz catalana ${ }^{13}$. Estamos, por tanto, ante una fragmentación por elusión del uso político de los medios informativos, algo que -lo hemos visto más arriba- también sucede entre la población poco interesada por la política (Prior, 2007). Sin embargo, al menos en el caso de CiU, por ser la formación política con grupo parlamentario propio con más representantes en el Congreso español, es este un hecho que llama la atención, al tiempo que muy probablemente no sea sino una muestra del enfoque fuertemente regionalista que imprime a sus tareas parlamentarias en Madrid. Por otro lado, también los políticos de ERC e ICV consumen en menor grado medios suprarregionales que los políticos del PSC y del PP (Tabla 5).

Entre los parlamentarios del PP y del PSC encontramos otra manifestación de la polarización, en este caso en forma de una fragmentación ideológica latente en su consumo de ofertas informativas mediáticas. Nuestros datos indican que la mayoría de parlamentarios que pertenecen a esas fracciones, de orientación más suprarregional, usan sobre todo medios periodísticos que les son políticamente cercanos. El 86\% de los políticos del PSC lee El País, un diario ubicado en la izquierda del espectro publicístico, y el $81 \%$ escucha la Cadena SER, posicionada, en ese mismo espectro izquierda-derecha, en un lugar muy parecido al de El País. La 1, cadena cuya audiencia entre los parlamentarios catalanes está por encima de la media, también puede ser incluida en esa fragmentación ideológica. En el momento en que se hizo la encuesta, el partido que gobernaba en Madrid era el PSOE. Atendiendo a la familiaridad ideológica que, según hemos visto ya, en España existe entre los medios públicos y los go-

${ }^{13}$ A pesar de ser un partido catalán independiente, el PSC forma parte de una federación de matriz española. Sus raíces históricas son la suma de muchas sensibilidades políticas, entre ellas algunas de matriz española (Reventós et al., 1998). 
biernos políticos, no resulta chocante que fueran los parlamentarios del PSC los que mostraran una mayor exposición a esa cadena de televisión suprarregional.

En cambio, los parlamentarios del PP preferían leer El Mundo (85\%) o La Razón (57\%), es decir, periódicos que son una correspondencia de su posición conservadora en el espectro publicístico-político. Esos mismos políticos recurrían con una frecuencia que estaba por encima de la media a las noticias de Antena $3(57 \%)$ o de Intereconomía (29\%). Ambas cadenas de televisión fueron ubicadas por el conjunto de los parlamentarios en el tramo conservador del espectro político izquierda-derecha.

Es bastante claro, en definitiva, que, en lo tocante a la exposición de los parlamentarios catalanes a la información de los medios periodísticos suprarregionales, se constata la existencia de dos formas distintas de fragmentación: una por elusión y otra por orientación ideologizada en el consumo. La combinación de ambos tipos de fragmentación es muy probable que esté conduciendo a una polarización de la imagen que los parlamentarios poseen de las cuestiones políticas en curso.

\section{Conclusiones}

Aunque hemos investigado el caso de un único Parlamento, los resultados obtenidos son sencillos de enmarcar en el contexto de otros estudios internacionales. El uso intensivo que los políticos hacen de los medios periodísticos es algo característico de los sistemas democráticos de tipo representativo. En ellos la idea de representación geográfica se suele traducir en un uso de medios regionales.

En nuestro caso, solo ha sido posible observar una polarización notable de tipo ideológico en el uso de las ofertas mediáticas informativas suprarregionales. Los diputados del Parlamento catalán se informan sobre todo a través de diarios y programas de televisión suprarregionales que refuerzan su posición política y, solo de manera limitada, mediante diarios con una línea editorial que diverge de ella. Este uso polarizado que los parlamentarios catalanes hacen de la información periodística es un fenómeno paralelo al que se da entre la población. De seguir avanzando, puede acabar dando lugar a un escenario en el que la democracia quede amenazada por el peligro de la desintegración, un escenario en el que los actores políticos, antes que abrirse a perspectivas o argumentos nuevos, buscan reforzar su posición exponiéndose solo a aquel periodismo que apoyan las opiniones y percepciones que ya tienen.

A nivel regional, sin embargo, la polarización se percibe solo en un grado menor. En su consumo de ofertas regionales de información, los diputados se centran en los medios generalmente percibidos como líderes -La Vanguardia o TV3, por ejemplo-, independientemente de la coloración partidista exacta que tenga su línea editorial.

Cabe valorar estos resultados desde la perspectiva que nos ha abierto la reflexión en torno a la esfera pública democrática. Si en un plano regional existen ofertas periodísticas usadas generalizadamente, son estas las primeras que podrían estar en disposición de fomentar la cohesión social, en caso de que se propongan ejercer de foro plural que posibilite el debate y la propuesta de soluciones. Servirían, entonces, para acercar a la sociedad al tipo ideal de esfera pública que hemos expuesto al comienzo del artículo. En caso de que esos medios lograran realizar una cobertura informativa variada y plural, el peligro de fragmentación -y, por tanto, de polarización del com- 
bate político- sería menor. Esta forma de informar por parte de los medios periodísticos y de consumir información por parte de los políticos sería compatible con el tipo ideal de esfera pública que defiende el modelo liberal-representativo. Por el contrario, los resultados que hemos obtenido en el nivel suprarregional, sí que han de ser considerados como una amenaza potencial para el funcionamiento de una democracia representativa. La exposición a coberturas informativas unilaterales conduce a una fragmentación de la esfera pública política. El escaso conocimiento de los argumentos de la parte contraria es un obstáculo para un sistema que basa su funcionamiento en las decisiones por mayoría.

\section{Referencias bibliográficas}

BARRERA, Carlos (2004): "Los medios de comunicación en España", en BARRERA, Carlos (coord.): Historia del periodismo universal. Barcelona, Ariel, pp. 285-318.

BAUM, Matthew A. \& GROELING, Tim (2008): "New Media and the Polarization of American Political Discourse". Political Communication, vol. XXV, $\mathrm{n}^{\circ} 4$, pp. 345-365. http://dx.doi.org/10.1080/10584600802426965

BECK, Ulrich \& SOPP, Peter (ed., 1997): Individualisierung und Integration. Neue Konfliktlinien und neuer Integrationsmodus?. Opladen, Leske + Budrich.

BENNETT, W. Lance \& IYENGAR, Shanto (2008): “A New Era of Minimal Effects? The Changing Foundations of Political Communication". Journal of Communication, vol. 58, no 4, pp. 707-731. http://dx.doi.org/10.1111/j.1460-2466.2008.00410.x

BERGANZA CONDE, María Rosa, et al. (2011): "La prensa gratuita ante las elecciones generales de España del 9 de marzo de 2008: cobertura, politización y frames en contraposición a la prensa de pago". Comunicacion y Sociedad, vol. XXIV, $\mathrm{n}^{\mathrm{o}} 1$, pp. 35-61.

BERRIO, Jordi (2000): La comunicació en democracia. Una visió sobre l'opinió pública. Bellaterra (Barcelona), Universitat Autònoma de Barcelona. Servei de Publicacions.

CONDE, Jordi (2002): "La regulación de los medios audiovisuales. Criterios técnicos y decisiones políticas. La posición de los diversos poderes territoriales", en TORNO MAS, Joaquín coord.: Democracia y medios de comunicación. Valencia, Tirant Lo Blanch, pp. 88-116.

CORTIÑAS ROVIRA, Sergi y PONT SORRIBES, Carles (2009): "Premsa diària i comunicación política. La percepció del projecte de reforma de l'Estatut de Catalunya en dotze diaris espanyols". Anàlisi, n ${ }^{\circ} 38$, pp. 117-134.

DADER, José Luis (2012): "Periodismo político y política del periodismo: Imaginando un futuro digno y sostenible", en BERROCAL GONZALO, Salomé y CAMPOS DOMÍNGUEZ, Eva (coords): La investigación en periodismo político en el entorno de los nuevos medios. Madrid, Sociedad Española de Periodística, pp. 35-58. 
DE MIGUEL, Juan Carlos y POZAS, Víctor (2009): “¿Polarización ideológica o económica? Relaciones entre los medios y el poder político y corporativo". Viento Sur, ${ }^{\circ} 103$, pp. 43-52.

DONSBACH, Wolfgang (1991a): Medienwirkung trotz Selektion. Einflussfaktoren auf die Zuwendung zu Zeitungsinhalten. Köln, Böhlau.

DONSBACH, Wolfgang (1991b): "Exposure to Political Content in Newspapers: The Impact of Cognitive Dissonance on Readers' Selectivity". European Journal of Communication, vol. 6, $\mathrm{n}^{\mathrm{o}}$ 2, pp. 155-186. http://dx.doi.org/10.1177 /0267323191006002003

FARIAS BATLLE, Pedro (2011): Informe anual de la profesión periodística. Madrid, Asociación de la Prensa de Madrid.

FERREE, Myra Marx et al. (2002): "Four models of the public sphere in modern democracies". Theory and Society, vol. 31, n 3, pp. 289-324.

GERHARDS, Jürgen y NEIDHARDT, Friedhelm (1991): "Strukturen und Funktionen moderner Öffentlichkeit. Fragestellungen und Ansätze", en MÜLLERDOOHM, Stefan y NEUMANN-BRAUN, Klaus eds.: Öffentlichkeit, Kultur, Massenkommunikation. Beiträge zur Medien- und Kommunikationssoziologie. Oldenburg, Universitätsverlag, pp. 31-89.

HALLIN, Daniel C. y MANCINI, Paolo (2004): Comparing Media Systems. Three Models of Media and Politics. New York, Cambridge University Press.

HOLLANDER, Barry A. (2008): "Tuning Out or Tuning Elsewhere? Partisanship, Polarization, and Media Migration from 1998-2006". Journalism \& Mass Communication Quarterly, vol. 85, $\mathrm{n}^{\circ}$ 1, pp. 23-40.

JANDURA, Olaf y MEYEN, Michael (2010): "Warum sieht der Osten anders fern? Eine repräsentative Studie zum Zusammenhang zwischen sozialer Position und Mediennutzung". Medien \& Kommunikationswissenschaft, vol. 58, $\mathrm{n}^{\circ}$ 2, pp. 208-226.

JARREN, Otfried y DONGES, Patrick (2006): Politische Kommunikation in der Mediengesellschaft. Eine Einführung. Wiesbaden, VS Verlag.

JORDANA, Jacint (1999): "Sindicalisme i política", en CAMINAL BADIA, Miquel y MATAS DALMASES, Jordi: El Sistema Politic de Catalunya. Barcelona, Tecnos, pp. 123-141.

KEPPLINGER, Hans Mathias (1998): Die Demontage der Politik in der Informationsgesellschaft. Freiburg, Karl Alber.

KEPPLINGER, Hans Mathias y MARX, Dorothea (2008): "Wirkungen und Rückwirkungen der politischen Kommunikation. Reziproke effekte auf Landtagsabgeordnete", en SARCINELLI, Ulrich y TENSCHER, Jens eds.: Politikherstellung und Politikdarstellung. Beiträge zur Politischen Kommunikation. Köln, Halem, pp. 188-208.

KSIAZEK, Thomas B. et al. (2010): "News-seekers and Avoiders: Exploring Patterns of Total News Consumption Across Media and the Relationship to Civic Partici- 
pation". Journal of Broadcasting \& Electronic Media, vol. 54, nº 4, pp. 551-568. http://dx.doi.org/ 10.1080/08838151.2010.519808

LEE, Jae Kook (2007): “The Effect Of The Internet On Homogeneity Of The Media Agenda: A Test of The Fragmentation Thesis". Journalism \& Mass Communication Quarterly, vol. 84, $\mathrm{n}^{\mathrm{o}}$ 4, pp. 745-760.

LIÑEIRA, Robert y PARDOS-PRADO, Sergi (2009): “Actors col-lectius: associacions, partits i mitjans de comunicacio", en ANDUIZA, Eva ed.: Informe sobre l'Estat de la Democrácia a Catalunya 2007. Barcelona, Fundació Jaume Bofill, pp. 245-319.

LÓPEZ GARCÍA, Guillermo (2009): Comunicación electoral y opinión pública. Valencia, Universidad de Valencia.

MUÑOZ-ALONSO, Alejandro y ROSPIR, Juan Ignacio (1999): Democracia mediática y campañas electorales. Barcelona, Ariel.

MUTZ, Diana C. (2006): Hearing the Other Side. New York, Cambridge University Press.

NIEDERMAYER, Oskar (2005): Bürger und Politik: Politische Orientierungen und Verhaltensweisen der Deutschen. Wiesbaden, VS Verlag.

PALACIO, Manuel (2001): Historia de la televisión en España. Barcelona, Gedisa.

PATTERSON, Thomas E. (1994): Out of order. New York, Vintage Books.

PETER, Susanne (1998): Expertenurteile über ausgewählte Print- und TV-Medien. Tesina de licenciatura no publicada. Departamento de Ciencias Sociales de la Universidad Johannes Gutenberg de Mainz (JGU).

PITKIN, Hanna Fenichel (1967): The concept of representation. Berkeley, University of California Press.

PRIOR, Markus (2007): Post-broadcast democracy. How media choice increases inequality in political involvement and polarizes elections. Cambridge, Cambridge University Press.

PUHE, Henry y WÜRZBERG, H. Gerd (1989): Lust \& Frust. Das Informationsverhalten des Deutschen Abgeordneten. Köln, Informedia.

REVENTÓS, Joan et al. (1998): Vint anys d'història del PSC. Barcelona, Fundació Rafael Campalans.

RODRÍGUEZ DÍAZ, Raquel et al. (2012): "Medios, partidos y confrontación en España: elecciones europeas 2009". Anàlisi, n 45, pp. 33-53.

RODRÍGUEZ-VIRGILI, Jordi et al. (2011): "La percepción pública de los políticos, los partidos y la política, y uso de medios de comunicación". Comunicación y Sociedad, vol. XXIV, nº 2, pp. 7-39.

SEYMOUR-URE, Collin (1974): The political impact of mass media. London, Constable. 
SLEMBECK, Tilman C. (1988): "Medienkonsum politischer Entscheidungsträger. Eine Untersuchung am Beispiel der Schweizer Bundesversammlung". Publizistik, vol. $33, n^{\circ} 4$, pp. 645-650.

STROUD, Natalie Jomini (2010): "Polarization and Partisan Selective Exposure". Journal of Communication, vol. 60, $\mathrm{n}^{\mathrm{o}} 3$, pp. 556-576. http://dx.doi.org/10.1111 /j.1460-2466.2010.01497.x

TRAPPEL, Josef et al. (eds., 2011): The Media for Democracy Monitor. A Cross National Study of Leading News Media. Göteborg, Nordicom.

VAN KEMPEN, Hetty (2007): "Media-Party Parallelism and Its Effects: A Cross-National Comparative Study". Political Communication, vol. 24, nº 3, pp. 303-320.

WENDELIN, Manuel (2011): Medialisierung der Öffentlichkeit. Kontinuität und Wandel einer normativen Kategorie der Moderne. Köln, Halem.

WESSLER, Hartmut (1999): Öffentlichkeit als Prozeß. Deutungsstrukturen und Deutungswandel in der deutschen Drogenberichterstattung. Opladen, Westdeutscher Verlag. 